\title{
Biologia e tabela de vida de Tetranychus desertorum (Acari: Tetranychidae) sobre folhas de feijão (Phaseolus vulgaris)
}

\author{
Edgar Rivero ${ }^{1} \&$ Carlos Vásquez ${ }^{2}$
}

\begin{abstract}
${ }^{1}$ Empresa Mixta Socialista de Arroz. Oficina Arroz del ALBA. Edifício INTI. Barquisimeto, Estado Lara, Venezuela.
2 Departamento de Ciencias Biológicas, Decanato de Agronomía, Universidad Centroccidental Lisandro Alvarado. Cabudare, Estado Lara, Venezuela. E-mail: carlosvasquez@ucla.edu.ve
\end{abstract}

\begin{abstract}
Biology and life table of Tetranychus desertorum (Acari: Tetranychidae) on leaves of kidney bean (Phaseolus vulgaris). Biological aspects and life table of the red spider mite, Tetranychus desertorum Banks, 1900, were studied on leaf discs of kidney bean (Phaseolus vulgaris Linnaeus) cultivar "Tacarigua" under laboratory conditions $\left(28 \pm 2^{\circ} \mathrm{C}, 70 \pm 10 \%\right.$ R.H. and 12:12h). Our results showed that total developmental time was 6.8 days for females, with partial duration of immature stages corresponding to 3.8, 1.4, 1.0 and 0.7 for egg, larva, protonymph and deutonymph, respectively. Preoviposition, oviposition and postoviposition periods were 1.1, 8.4 and 1.3 days, respectively; and the higher mean fecundity (6.93 eggs/female/day) was observed on day 4 . Female mean longevity was 10 days. The life table parameters recorded were: net reproduction rate $(R o)=41.10$ individuals; generation time $(T)=11.15$ days; intrinsic natural growth $\left(r_{m}\right)=$ 0.144 individuals/female/day, and finite natural increase rate $(\lambda)=1.155$ individuals/female. Our findings could be a basis for further studies devoted to determine damage and control strategies for T. desertorum on kidney bean crops.
\end{abstract}

KEY WORDS. Fecundity; life history; red spider mite; tetranychid.

As espécies de Tetranychus Dufour, 1832 apresentam ampla distribuição geográfica (Bolland et al. 1998) e alto potencial biológico, o qual depende da planta hospedeira, das condições ambientais (principalmente temperatura e umidade), das práticas culturais utilizadas e dos aspectos intrínsecos de cada espécie de ácaro (Gutierrez \& Helle 1985, Tomczyk \& KRopCZyNSKA 1985, WRENSCH 1985).

Além do efeito da temperatura sobre o tempo de desenvolvimento, a fecundidade e a longevidade dos tetraniquídeos (Hazan et al. 1973, Carey \& Bradley 1982, Biswas et Al. 2004, PRASLICKA \& Huszár 2004), as características morfológicas e químicas das plantas hospedeiras podem exercer influência nos parâmetros biológicos destes ácaros (Crooker 1985). Vásquez et al. (2007) observaram algumas características de antibiose no cultivar de feijão (Phaseolus vulgaris Linnaeus) ICA-Pijao ao ataque de Tetranychus cinnabarinus (Boisduval, 1987), uma vez que fêmeas dessa espécie mostraram menor oviposição e sobrevivência nesse cultivar do que nos cultivares Coche e Tucupita, sugerindo que ICA-Pijao poderia produzir certos compostos de anti-alimentação para os tetraniquídeos. $\mathrm{Na}$ Venezuela, Tetranychus urticae Koch, 1836 e Tetranychus ludeni Zacher, 1913 têm sido observados produzindo redução significativa no crescimento de plantas de feijão quando essas são atacadas por densidades superiores a 1,63 ácaros/cm² (APONTE \& Aponte 1990, Morros \& Aponte 1995).

O ácaro-vermelho, Tetranychus desertorum Banks, 1900 tem sido relatado em 173 espécies de plantas e encontra-se distribu- ído na Argentina, Brasil, China, Estados Unidos, Hawai, México e Venezuela, entre outros países (Bolland et al. 1998), podendo causar danos ao feijoeiro semelhantes àqueles relatados para $T$. urticae (Moraes \& Flechtmann 2008). Estudos prévios na Venezuela têm demonstrado que plantas de feijão são susceptíveis ao ataque de T. desertorum sob condições de irrigação (DOREsTe 1984).

Na Venezuela, T. desertorum encontra-se frequentemente associado à cultura de feijão, porém não existe informação sobre a biologia nem os danos produzidos por esta espécie de ácaro. Neste trabalho foram estudados alguns aspetos biológicos e dados da tabela de vida de $T$. desertorum criados em folhas de feijão cultivar "Tacarigua", a fim de que sejam estabelecidas bases úteis para futuras pesquisas para determinar o impacto desse ácaro na produção de feijão.

\section{MATERIAL E MÉTODOS}

Coleta, determinação da espécie e criação-estoque

Fêmeas e machos de $T$. desertorum foram coletados em plantas de Convolvulus sp. (Convolvulaceae) em El Jebe, ao norte de Barquisimeto, Município Iribarren, do Estado Lara, Venezuela. As amostras de folhas infestadas com ácaros foram acondicionadas em sacos plásticos e levadas ao laboratório de Zoología Agrícola, Decanato de Agronomia, Universidad Centroccidental Lisandro Alvarado.

No laboratório, lâminas contendo fêmeas e machos foram preparadas usando Meio de Hoyer para observação ao microscópio ótico (Moraes \& Flechtmann 2008). A espécie foi deter- 
minada com base em caracteres morfológicos (PRITCHARD \& BAKER 1955). Os espécimes vouchers foram depositados no laboratório de Zoologia Agrícola (MZ-UCLA228, 5 machos e 2 fêmeas, MZUCLA229 5 machos e 1 fêmeas sobre Convolvulus sp., Via El Jebe, Barquisimeto, estado Lara, fevereiro 2008, A. Sivira leg.).

Após a confirmação da espécie, plantas de feijão cultivar "Tacarigua" com aproximadamente 40 dias de idade cultivadas em sacos de polietileno $(19 \times 22 \mathrm{~cm})$ foram artificialmente infestadas com os ácaros para assim garantir uma criação-estoque de indivíduos para o ensaio.

\section{Ciclo de vida}

Para a obtenção de indivíduos de idade homogênea, previamente ao início do ensaio de biologia, prepararam-se cinco unidades de criação segundo a metodologia proposta por HeLle \& Overmeer (1985). Cada unidade de criação consistiu em uma placa de Petri (9 $\mathrm{cm}$ de diâmetro) contendo uma espuma de poliuretano circular de 1,0 cm de espessura umedecida com água destilada. Sobre cada unidade foi acondicionado um disco de folha de feijão cultivar "Tacarigua" (3 cm de diâmetro) sobre o qual foram colocadas cinco fêmeas e um macho para promover o acasalamento e assim possibilitar a produção de ovos. Após 24 horas, as fêmeas e os machos foram descartados e o número de ovos foi registrado. Os ovos foram deixados nessas unidades de criação-estoque e observados até o surgimento dos adultos, os quais foram, então, utilizados no estudo do ciclo biológico.

O ciclo biológico de $T$. desertorum foi estudado em laboratório ( $28 \pm 2^{\circ} \mathrm{C}, 70 \pm 10 \%$ U.R. e $12: 12 \mathrm{~h}$ de fotofase) usando unidades de criação como as descritas acima. O estudo foi iniciado com 30 unidades de criação, cada uma delas contendo três discos de folhas de feijão (3 cm de diâmetro) com a superfície adaxial dos discos voltada para cima; os discos foram margeados com uma faixa úmida de algodão para evitar o escape dos ácaros e manter a turgidez das folhas. Sobre cada disco de folha foram colocados uma fêmea e um macho com um dia de idade provenientes da criação-estoque estabelecida em laboratório (como descrito acima).

As unidades de criação foram observadas a cada uma hora, ao microscópio estereoscópico, para observação do momento da postura, após o qual as fêmeas foram removidas. Para se determinar o tempo de incubação dos ovos e a duração das fases imaturas (larva, protoninfa e deutoninfa), as observações foram realizadas a cada 12 horas. Nos casos em que os discos de folha perderam a turgidez, os ácaros foram transferidos para novas unidades de criação.

\section{Pré-oviposição, oviposição, pós-oviposição e longevidade}

Os períodos de pré-oviposição, oviposição e pósoviposição foram estudados num grupo de 30 fêmeas acasaladas $\left(28 \pm 2^{\circ} \mathrm{C}, 70 \pm 10 \%\right.$ U.R. e 12:12h). Cada fêmea com 2-3 dias de idade foi colocada junto com um macho em uma unidade de criação isolada, a qual foi observada em intervalos de 12 horas. Os dados foram expressos como número médio de dias para cada período (pré-oviposição, oviposição e pós-oviposição).

\section{Tabela de vida}

O cálculo da tabela de vida de T. desertorum foi feito seguindo a metodologia proposta por BIRCH (1948). Os parâmetros populacionais considerados foram: taxa líquida de reprodução (Ro), duração média de uma geração (T), razão intrínseca de aumento $\left(\mathrm{r}_{\mathrm{m}}\right)$ e razão finita de aumento $(\lambda)$, os quais foram estimados com base nos dados de oviposição e sobrevivência obtidos nos ensaios anteriores.

Segundo Rabinovich (1980) estes parâmetros são definidos como:

a) Razão intrínseca de aumento $\left(\mathrm{r}_{\mathrm{m}}\right)$ : capacidade de multiplicação de uma população em uma geração:

$$
\left.\sum_{x=0}^{\infty} e^{-r m_{x}}\right|_{x} m_{x}
$$

onde: $\mathrm{x}=$ idade dos indivíduos em dias, $\mathrm{l}_{\mathrm{x}}=$ proporção de indivíduos vivos à idade " $\mathrm{x}$ ", $\mathrm{m}_{\mathrm{x}}=$ número de descendentes produzidos por cada fêmea no intervalo de idade " $\mathrm{x}$ "

b) Duração média de uma geração (T): representa o tempo médio entre duas gerações sucessivas:

$$
T=\frac{\sum x l_{x} m_{x}}{\sum I_{x} m_{x}}
$$

c) Taxa líquida de reprodução $\left(\mathrm{R}_{0}\right)$ : conhecida como tempo de substituição, reflete o número médio de descendentes que cada fêmea da população é capaz de produzir durante sua vida:

$$
R_{0}=\sum_{x=0} I_{x} m_{x}
$$

d) Razão finita de aumento (l): é uma taxa populacional finita e não-instantânea, interpretada como o número de indivíduos que se adiciona à população por cada indivíduo e por unidade de tempo: $\lambda=e^{r_{m}}$

\section{RESULTADOS E DISCUSSÃO}

\section{Duração do ciclo de vida}

O tempo total médio de desenvolvimento de T. desertorum de ovo a adulto sobre folhas de feijão cultivar "Tacarigua" foi estimado em 6,8 dias (com mínimo de seis e máximo de sete dias). A fase de ovo durou em média 3,8 dias e as fases imaturas (larva, protoninfa e deutoninfa) 1,4, 1,0 e 0,7 dias, respectivamente (Tab. I). Resultados similares foram obtidos por PraslickA \& Huszár (2004), os quais determinaram um tempo de desenvolvimento de 6,90 dias para $T$. urticae criado sobre folhas de $P$. vulgaris à temperatura de $30^{\circ} \mathrm{C}$. Possivelmente as semelhanças entre esses estudos sejam devidas aos efeitos causados tanto pela planta hospedeira como pela temperatura, pois estudos prévios têm indicado, por um lado, que as folhas de feijão constituem o melhor substrato para a criação dos tetraniquídeos por diminuir o tempo requerido para completar o ciclo bioló- 
gico (Helle \& Overmeer 1985). Por outro lado, temperaturas superiores a $25^{\circ} \mathrm{C}$ produzem diminuição do tempo de desenvolvimento e incremento de fecundidade destes ácaros (TAnigoshi et al. 1975, Aponte \& McMurtry 1997). De forma semelhante, VASCONCELos et al. (2004) demostraram que o tempo de desenvolvimento de Tetranychus abacae Baker \& Pritchard sobre folhas de Musa sp. diminuiu em 33,2\% quando a temperatura de criação incrementou de 25 a $30^{\circ} \mathrm{C}$. Assim, outras espécies de Tetranychus necessitaram de maior tempo para completar o ciclo de vida à medida que as temperaturas do ensaio decresceram. Assim, o tempo requerido por T. ludeni para completar o ciclo de vida foi de 9,98 dias para as fêmeas quando estas foram criadas sobre folhas de feijão a temperaturas de $26,34^{\circ} \mathrm{C}$ (Morros \& Aponte 1994), enquanto que Tetranychus marianae McGregor completou o ciclo de vida em 10,73 dias sobre folhas de maracujá à temperatura de $25^{\circ} \mathrm{C}$ (NorONHA 2006).

Tabela I. Duração (dias) e sobrevivência (\%) das fases de desenvolvimento de $T$. desertorum criados $(\mathrm{N}=30)$ sobre folhas de feijão (P. vulgaris) cultivar Tacarigua a $28 \pm 2^{\circ} \mathrm{C}, 70 \pm 10 \%$ U.R. e 12:12 h, em laboratório.

\begin{tabular}{lcc}
\hline \multicolumn{2}{l}{ Fase de desenvolvimento } & Tempo médio \pm E.P. Sobrevivência (\%) \\
\hline Ovo & $3,8 \pm 0,22$ & 100 \\
Larva & $1,4 \pm 0,14$ & 97 \\
Protoninfa & $1,0 \pm 0,17$ & 95 \\
Deutoninfa & $0,7 \pm 0,13$ & 91 \\
Ovo-adulto & $6,8 \pm 0,35$ & \\
\hline
\end{tabular}

\section{Tempo de pré-oviposição, oviposição e pós-ovipo- sição, e longevidade de $T$. desertorum}

A duração média do período de pré-oviposição foi de 1,1 dias, enquanto que os períodos de oviposição e pós-oviposição foram de 8,4 e 1,3 dias, respectivamente (Tab. II). Em relação ao número de ovos, o maior número de ovos (208) foi registrado no quarto dia, correspondendo a uma taxa de oviposição de 6,93 ovos/fêmea/dia ${ }^{-1}$. Após o oitavo dia, observou-se uma considerável diminuição na quantidade de ovos/dia (Fig. 1). Outras espécies de Tetranychus têm mostrado valores de fecundidade similares

Tabela II. Duração (dias) dos períodos de pré-oviposição, oviposição e pós-oviposição, e longevidade de $T$. desertorum criados $(\mathrm{N}=30)$ sobre folhas de feijão ( $P$. vulgaris) cultivar Tacarigua a $28 \pm 2^{\circ} \mathrm{C}, 70 \pm 10 \%$ U.R. e 12:12 h, em laboratório.

\begin{tabular}{cc}
\hline Períodos & Tempo (dias) \pm E.P. \\
\hline Pré-oviposição & $1,1 \pm 0,35$ \\
Oviposição & $8,4 \pm 1,91$ \\
Pós-oviposição & $1,3 \pm 0,47$ \\
Longevidade & $10,0 \pm 0,71$ \\
\hline
\end{tabular}

às encontradas neste estudo. Morros \& APONTE (1994) encontraram que a fecundidade de T. ludeni quando foi criado em folhas de feijão foi de 118,09 ovos/fêmea, a qual resultou em uma taxa de oviposição de 7,90 ovos/fêmea/dia. Além disso, PrasLicKa \& HusZÁr (2004) obtiveram valores de fecundidade entre 89,00 e 92,90 para $T$. urticae criado em folhas de feijão a 25 e $30^{\circ} \mathrm{C}$, respectivamente. Por outro lado, NorOnHa (2006) observou que $T$. marianae mostrou valores de fecundidade (75,46 ovos/fêmea) e taxa de oviposição (3,69 ovos/fêmea/dia) relativamente inferiores quando foi criado sobre folhas de maracujá. As semelhanças observadas nos parâmetros reprodutivos destas espécies podem ser explicadas pelo efeito da planta hospedeira uma vez que a composição química da planta pode influenciar fecundidade, mortalidade e desenvolvimento dos estádios imaturos (CROOKER 1985).

Com relação à longevidade, o tempo de vida das fêmeas de $T$. desertorum (10,0 dias) foi inferior aos dados registrados para T. ludeni (18,2 dias) criado sobre folhas de feijão (Morros \& Aponte 1994) e para T. marianae (24,53 dias), criado sobre folhas de maracujá (NoRONHA 2006).

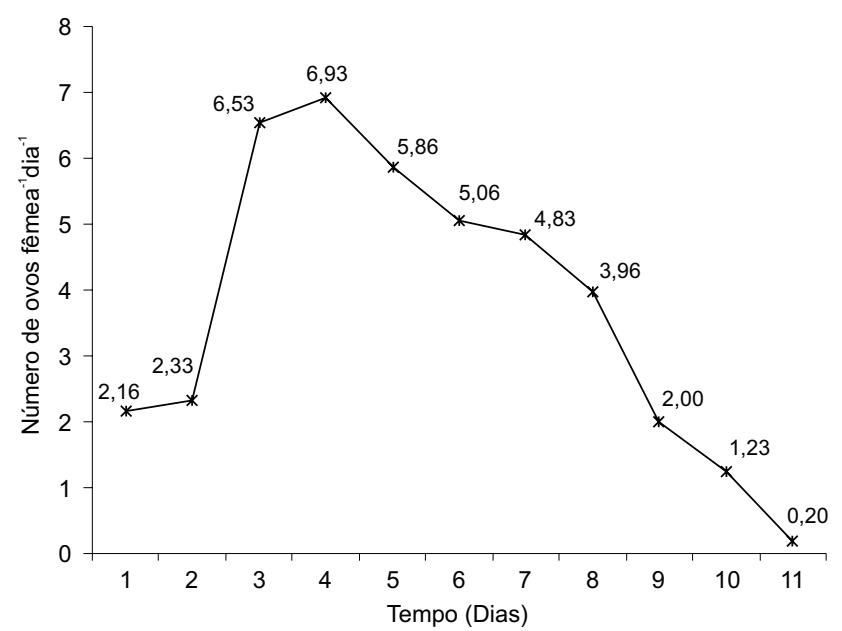

Figura 1. Taxa de oviposição de $T$. desertorum em folhas de feijão (P. vulgaris) cultivar "Tacarigua" a $28 \pm 2^{\circ} \mathrm{C}, 70 \pm 10 \%$ U.R. e 12:12h (média de 30 observações), em laboratório.

\section{Tabela de vida}

A sobrevivência $\left(1_{x}\right)$ de $T$. desertorum foi máxima durante os primeiros 10 dias e foi mantida acima de $50 \%$ até o $15^{\circ}$ dia, diminuindo gradualmente até o $17^{\circ}$ dia. Além disso, a produção de descendentes/fêmea/dia $\left(\mathrm{m}_{\mathrm{x}}\right)$ foi relativamente constante no período do nono ao $14^{\circ}$ dia e, após esse período, diminuiu até atingir valores mínimos no $17^{\circ}$ dia (Fig. 2).

Os parâmetros da tabela de vida obtidos para T. desertorum foram os seguintes: taxa líquida de reprodução $(\mathrm{Ro})=41,10$ indivíduos; duração média de uma geração $(\mathrm{T})=11,15$ dias; razão intrínseca de crescimento $\left(\mathrm{r}_{\mathrm{m}}\right)=0,14$ indivíduos/fêmea/dia, e, razão finita de aumento $(\lambda)=1,15$ indivíduos/fêmea (Tab. III). 


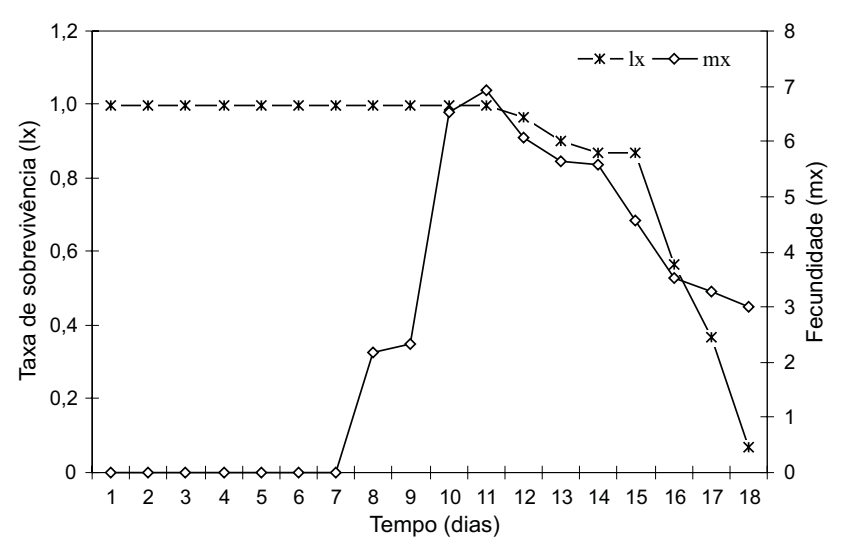

Figura 2. Taxa de sobrevivência e número de descendentes/fêmea/dia de $T$. desertorum criados em folhas de feijão ( $P$. vulgaris) cultivar "Tacarigua" a $28 \pm 2^{\circ} \mathrm{C}, 70 \pm 10 \%$ U.R. e 12:12 h, em laboratório.

Tabela III. Parâmetros populacionais de T. desertorum criados em folhas de feijão (P. vulgaris) cultivar Tacarigua a $28 \pm 2^{\circ} \mathrm{C}, 70 \pm 10 \%$ U.R. e 12:12 h.

\begin{tabular}{cc}
\hline Parâmetros populacionais & Valores observados \\
\hline Razão intrínseca de aumento $(\mathrm{rm})$ & 0,144 \\
Duração média de uma geração $(\mathrm{T})$ & 11,150 \\
Taxa líquida de reprodução (R0) & 41,100 \\
Razão finita de aumento $(\lambda)$ & 1,155 \\
\hline
\end{tabular}

Os valores de rm e $\lambda$ obtidos para T. desertorum são similares aos obtidos para T. marianae $(0,172$ e 1,187 , respectivamente) em folhas de maracujazeiro (Noronha 2006), enquanto que os valores de rm e $\lambda$ foram maiores para T. ludeni $(0,253$ e 1,287 , respectivamente) quando criados em feijoeiro (Morros \& Aponte 1994) e para T. urticae (0,298 e 1,347, respectivamente) em folhas de pimentão (GAllardo et al. 2005). Segundo Gutierrez \& Helle (1985) os valores de $r_{m}$ dos tetraniquídeos pode ser maior quando a duração da geração é menor e a fecundidade é maior, porém também pode ser influenciado pela natureza da planta hospedeira, superfície disponível para cada individuo e os valores de temperatura e umidade.

Os resultados obtidos neste estudo constituem a primeira informação disponível sobre aspectos biológicos de $T$. desertorum e servem como base para futuras investigações para determinar o dano e as estratégias de controle de T. desertorum na cultura de feijão. Porém novos estudos são necessários para se determinar sua importância como praga na cultura de feijão e o impacto sobre a produção.

\section{AGRADECIMENTOS}

À Clarice Pinto Máximo Espíndola (Instituto Butantan) e aos revisores anônimos pela revisão e sugestões ao manuscrito.

\section{LITERATURA CITADA}

Aponte, O. \& J.A. McMurtry. 1997. Biology, life table and mating behavior of Oligonychus persea (Acari: Tetranychidae). International Journal of Acarology 23 (3): 199-207.

Aponte, O.R. \& F.A. Aponte. 1990. Daños de Tetranychus urticae Koch y su influencia en el cultivo de la caraota $P$. vulgaris $L$. Boletín de Entomología Venezolana 5 (20): 197-204.

BIRCH, L. 1948. The intrinsic rate of natural increase of an insect population. Journal of Animal Ecology 17: 15-26.

Biswas, G.C.; W. Islam; M.M. Haque; R.K. SAHA; K.M.F. Hoque; M.S. Islam \& M.E. Haque. 2004. Some biological aspects of carmine spider mite, Tetranychus cinnabarinus Boisd. (Acari: Tetranychidae) infesting egg-plant from Rajshahi. Journal of Biological Science, Bangladesh 4 (5): 588-591.

Bolland, H.R.; J. Gutierrez \& C.H.W. Fletchmann. 1998. World catalogue of the spider mite family (Acari: Tetranychidae). Leiden, Koninklijke Brill NV, 408p.

Carey, J. \& J. Bradley. 1982. Development rates, vital schedules, sex ratios, and life tables for Tetranychus urticae, T. turkestani and T. pacificus (Acarina: Tetranychidae) on cotton. Acarologia 23 (4): 333-345.

Crooker, A. 1985. Embryonic and juvenil development, p. 149163. In: W. Helle \& M. SAbelis (Eds). Spider mites: their biology, natural enemies and control. Amsterdam, Elsevier Science Publishers B.V., vol. 1A, 405p.

Doreste, E. 1984. Acarología. San José, Instituto Interamericano de Cooperación para la Agricultura, 391p.

Gallardo, A.; C. Vásquez; J. Morales \& J. Gallardo. 2005. Biología y enemigos naturales de Tetranychhus urticae en pimentón. Manejo Integrado de Plagas y Agroecologia 74: 34-40.

Gutierrez, J. \& W. Helle. 1985. Evolutionary changes in the Tetranychidae, p. 91-107. In: W. Helle \& M.W. SABelis (Eds). Spider mites: their biology, natural enemies and control. Amsterdam, Elsevier Science Publisher B.V., vol. 1A, 405p.

HaZAN, A.; U. Gerson \& A. TAHORI. 1973. Life history and life tables of the carmine spider mite. Acarologia 15 (3): 414-440.

Helle, W. \& W.P.J. Overmeer. 1985. Rearing techniques, p. 331335. In: W. Helle \& M.W. Sabelis (Eds). Spider mites: their biology, natural enemies and control. Amsterdam, Elsevier Science Publisher B.V., vol. 1A, 405p.

Moraes, G.J. de \& C.W. Flechtmann. 2008. Manual de acarologia: acarologia básica e ácaros de plantas cultivadas no Brasil. Ribeirão Preto, Holos, 288p.

Morros, M. \& O. Aponte. 1994. Biología y tabla de vida de Tetranychus ludeni Zacher en caraota. Agronomía Tropical 44 (4): 667-677.

Morros, M. \& O. Aponte. 1995. Efecto de dos niveles de infestación de Tetranychus ludeni Zacher sobre las fases de desarrollo de la caraota. I. Nivel de campo. Agronomía Tropical 54 (2): 189-194.

Noronha, A. 2006. Biological aspects of Tetranychus marianae McGregor (Acari: Tetranychidae) reared on yellow passion fruit (Passiflora edulis Sims f. flavicarpa Deg.) leaves. Revista 
Brasileira de Zoologia 23 (2): 404-407.

Praslicka, J. \& J. Huszár. 2004. Influence of temperature and host plants on the development and fecundity of the spider mite Tetranychus urticae (Acarina: Tetranychidae). Plant Protection Science 40 (4): 141-144.

Pritchard, E. \& E.W. BaKer. 1955. A Revision of the spider mites family Tetranychidae. San Francisco, California, Pacific Coast Entomological Society, vol. 2, 472 p.

RABINOVICH, J. 1980. Introducción a la ecología de poblaciones animales. México, Compañía Editorial Continental, 313p.

Tanigoshi, L.K.; S.C. Hoyt; R.W. Browne \& J.A. Logan. 1975. Influence of temperature on populations increase of Tetranychus mcdanieli (Acarina: Tetranychidae). Annals of Entomological Society of America 68: 972-978.

Tomczyк, A. \& D. Kropczynska. 1985. Effects on the host plant, p. 317-329. In: W. Helle \& M. Sabelis (Eds). Spider mites: their biology, natural enemies and control. Amsterdam, Elsevier Science Publishers B.V., vol. 1A, 405p.

Vasconcelos, G.J.N.; F.R. DA Silva; M.G.N. Gondim JR.; R. Barros \& J. Oliveira. 2004. Efeito de diferentes temperaturas no desenvolvimento e reprodução de Tetranychus abacae Baker \& Pritchard (Acari: Tetranychidae) em bananeira Musa sp. cv. prata. Neotropical Entomology 33 (2): 149-154.

Vásquez, C.; M. Colmenárez; N. Valera \& L. Díaz. 2007. Antibiosis of kidney beans cultivars to the carmine spider mite, Tetranychus cinnabarinus (Boisduval) (Acari: Tetranychidae). Integrated Control of Plant-feeding Mites. IOBC/wprs Bulletin 30 (5): 133-138.

WRENSCH, D.L. 1985. Reproductive parameters, p. 165-168. In: W. Helle \& M. Sabelis (Eds). Spider mites: their biology, natural enemies and control. Amsterdam, Elsevier Science Publishers B.V., vol. 1A, 405p.

Submitted: 04.VI.2008; Accepted: 05.III.2009.

Editorial responsibility: Pedro Gnaspini 